Enfermagem Brasil 2018;17(5):497-503

\title{
ARTIGO ORIGINAL A percepção de gestantes sobre os diferentes tipos de parto
}

Hévyllin Cipriano Rodrigues Félix*, Ana Carolina Lima Silva*, Thaís Gabriela da Cruz Matias*, Bibiane Dias Miranda, D.Sc. ${ }^{* *}$, Sueli Riul da Silva ${ }^{\star \star *}$, Mariana Torreglosa Ruiz**

${ }^{*}$ Enfermeira, Graduadas pelo curso de graduação em Enfermagem da Universidade Federal do Triângulo Mineiro (UFTM), ${ }^{* *}$ Professoras Adjunto do Curso de Graduação em Enfermagem da Universidade Federal do Triângulo Mineiro (UFTM), ${ }^{* \star *}$ Professora Associada do Programa de Pós-Graduação em Atenção à Saúde da Universidade Federal do Triângulo Mineiro (PPGAS UFTM)

Recebido em 9 de fevereiro de 2018; aceito em 25 de julho de 2018.

Endereço para correspondência: Mariana Torreglosa Ruiz, Praça Manoel Terra, 330 Câmpus I, Curso de Graduação em Enfermagem da Universidade Federal do Triângulo Mineiro, 38025-100 Uberaba MG, E-mail: Mariana Torreglosa Ruiz: marianatorreglosa@hotmail.com; Hévyllin Cipriano Rodrigues Félix: hevyllin_rodrigues@hotmail.com; Ana Carolina Lima Silva: aninhacarol.enf@gmail.com; Thais Gabriela da Cruz Matias: thais_gabriela_15@hotmail.com; Bibiane Dias Parreira Miranda: bibianedias@yahoo.com.br, Sueli Riul da Silva: sueliriul@terra.com.br.

\section{Resumo}

Introdução: A informação sobre os diferentes tipos de parto, riscos e benefícios são direitos universais das mulheres grávidas previstos pela Aliança Global para Maternidade Segura, e a preferência e escolha da mulher pelo tipo de parto devem ser consideradas sempre em posse deste conhecimento. Objetivo: Identificar a percepção de gestantes sobre diferentes tipos de parto. Material e métodos: Estudo transversal, a partir de entrevista com 208 gestantes assistidas entre dezembro de 2014 e fevereiro de 2015, em um ambulatório de um hospital de ensino, com aplicação de instrumento com questões fechadas e abertas, elaborado pelos pesquisadores e testado mediante estudo-piloto. Resultados: $54,8 \%$ relataram não ter recebido orientação; a maioria afirmou que o parto normal é mais seguro $(80,8 \%)$, recebia alta mais rápida (99\%), é mais cômodo/confortável (70,7\%), e o procedimento mais rápido (56,7\%); $47,1 \%$ acreditavam que o fórceps possui maior risco de morte para o recém-nascido e $51,9 \%$ relataram que a cesárea possui maior risco de complicações. Conclusão: Frente aos resultados e altos índices de cesáreas, identifica-se a necessidade de educação em saúde referente aos diferentes tipos de parto e ressalta-se a importância do enfermeiro neste processo, enquanto educador.

Palavras-chave: recém-nascido, cesárea, parto normal.

\begin{abstract}
The perception of pregnant women about the different types of delivery

Introduction: Information of the different types of childbirth, risks and benefits are universal rights of pregnant women provided by the Global Alliance for Safe Motherhood, and the preference and choice of the woman for the type of delivery should always be considered. Objective: To identify the perception of pregnant women about different types of delivery. Methods: A cross-sectional study, based on an interview with 208 pregnant women, assisted between December 2014 and February 2015, in an outpatient clinic of a teaching hospital, using an instrument with closed and open questions, tested by a pilot study. Results: $54.8 \%$ reported not receiving counseling; the majority said that normal childbirth is safer $(80.8 \%)$, hospital discharge faster $(99 \%)$, is more comfortable $(70.7 \%)$, and the fastest procedure $(56.7 \%) ; 47.1 \%$ believed that forceps had a higher risk of death for the newborn and $51.9 \%$ reported that cesarean section had a higher risk of complications. Conclusion: In view of the results and high rates of cesarean sections, the need for health education regarding the different types of delivery is identified and the importance of the nurse in this process as an educator is emphasized.
\end{abstract}

Key-words: infant newborn, cesarean section, natural childbirth. 


\section{Resumen \\ La percepción de gestantes sobre diferentes tipos de parto}

Introducción: La información sobre los diferentes tipos de parto, riesgos y beneficios son derechos universales de las mujeres embarazadas previstas por la Alianza Global para la Maternidad Segura, y preferencia y elección de la mujer por tipo de parto debe ser considerada siempre en posesión de este conocimiento. Objetivo: Identificar la percepción de gestantes sobre diferentes tipos de parto. Material y métodos: Estudio transversal, a partir de entrevista con 208 gestantes, asistidas entre diciembre de 2014 y febrero de 2015, en un ambulatorio de un hospital de enseñanza, con aplicación de instrumento con cuestiones cerradas y abiertas, elaborado por los investigadores y probado mediante estudio- piloto. Resultados: 54,8\% relató no haber recibido orientación; la mayoría afirmó que el parto normal: es más seguro $(80,8 \%)$, recibía alta más rápido (99\%), es más cómodo $(70,7 \%)$, y el procedimiento más rápido $(56,7 \%)$; $47,1 \%$ creía que fórceps tiene mayor riesgo de muerte para el recién nacido y $51,9 \%$ relató que cesárea tiene mayor riesgo de complicaciones. Conclusión: Frente a los resultados y altos índices de cesáreas, se identifica la necesidad de educación en salud referente a diferentes tipos de parto y se resalta la importancia del enfermero en este proceso, como educador.

Palabras-clave: recién nacido, cesárea, parto normal.

\section{Introdução}

A educação em saúde é uma importante ferramenta na assistência ao ciclo gravídicopuerperal, uma vez que a gravidez trata-se de um período delicado e gerador de dúvidas e ansiedade para a gestante e seus familiares [1]. Assim, o esclarecimento sobre tipos de parto deve iniciar durante o pré-natal, tendo em vista que assistência qualificada pode promover uma opção mais consciente e segura, favorecendo condições mais humanizadas do nascimento, tanto para a mãe quanto para o recém-nascido [2].

Dados do Inquérito Populacional Brasileiro "Nascer no Brasil" apontam que do total de partos investigados, apenas $5 \%$ foram ultimados por via vaginal sem intervenções; $48,1 \%$ por via vaginal com intervenções (uso de ocitócitos; manobra de Kristeller, amniotomia e episiotomia) e $51,9 \%$ por cesárea [3].

A Organização Mundial de Saúde (OMS) recomenda que a taxa de cesáreas não ultrapasse $15 \%$ de todos os partos [4] e, em declaração publicada em 2015 , sugere-se que não ultrapasse $10 \%$ [5]. A cesárea é uma intervenção efetiva para salvar vidas, quando indicada por motivos justificáveis, entretanto, taxas superiores a $10 \%$ não reduziram mortalidade, mas aumentaram índices de complicações [5].

Desde 2009, índices de cesárea no Brasil ultrapassaram 50\% das vias de parto, em ascensão crescente em relação ao parto vaginal [6]. Esta afirmativa pode ser corroborada por dados do Datasus, que apontam no ano de 2016 índice de $55,4 \%$ de cesáreas [7]. A prevalência de cesáreas realizadas pela saúde suplementar (convênios e particulares) alcança taxas muito mais elevadas que variam de 80 a $90 \%$ [8].

Para redução dos índices de cesárea, faz-se fundamental a comunicação no processo de educação em saúde, estabelecida entre profissional de saúde e as mulheres visando à promoção de atitudes mais conscientes e seguras. Contudo, não se pode esquecer que a comunicação para ser efetiva deve ser estabelecida também com a rede social desta, uma vez que relações com familiares e amigos têm forte influência nas escolhas e podem ou não favorecer clareza nos questionamentos e consequentemente gerar dúvidas relacionadas ao parto [9].

A comunicação profissional deve ser pautada nos seguintes aspectos: conhecimento dos diferentes tipos de parto, suas vantagens e desvantagens, informações sobre o processo fisiológico do parto e preparo para o momento do nascimento, além de possibilitar a reflexão de como esses aspectos podem influenciar a vida da mulher e a de seu filho [10].

Ressalta-se que a expectativa da mulher em relação ao parto pode mudar ao longo da gestação. Dados apontam que $66 \%$ das mulheres preferiam o parto vaginal no início da gestação, 27,6\% cesárea e, 6,1\% não tinham preferência definida. Já no final da gestação, apenas um terço das mulheres referiu já ter clara sua escolha quanto à via de parto, $51,5 \%$ optariam por cesárea e destas, $65,7 \%$ esperavam por cesárea eletiva previamente agendada [2].

De acordo com os mesmos autores, a escolha inicial pelo parto vaginal foi motivada pelo reconhecimento do processo fisiológico do nascimento e pela crença na recuperação pós- 
parto mais rápida. Para multíparas, a experiência anterior de parto normal positiva influenciou fortemente esta opção [2].

Entre mulheres que tiveram como primeira opção a cesárea, o motivo alegado foi o medo sentido em relação a "sentir dor" e práticas não humanizadas. Também foram significantes as alegações: maior comodidade; acreditar ser a via mais segura para o recém-nascido, ter tido intercorrências/problemas de saúde na gestação e preferência do marido por esta via. Enfatizase a partir destas justificativas a necessidade de mudança dos modelos assistenciais e a importância do papel educativo dos profissionais de saúde [2].

A informação sobre tipos de parto, riscos e benefícios são direitos universais das mulheres grávidas previstos pela Aliança Global para Maternidade Segura e a preferência e escolha da mulher pelo tipo de parto deve ser considerada sempre em posse deste conhecimento.

Dados os altos índices de cesárea, suas possíveis complicações e a necessidade de orientação sobre os diferentes tipos de parto durante a gestação, incluindo suas vantagens e desvantagens, identificar a percepção das gestantes acerca da temática é pertinente e relevante, justificando a execução deste estudo. Ressalta-se que a partir dos resultados, podese 'repensar' a assistência pré-natal e a forma de educação em saúde, adequando para as diferentes realidades institucionais.

Este estudo teve por objetivo identificar a percepção de gestantes sobre os diferentes tipos de parto.

\section{Material e métodos}

Trata-se de um estudo descritivo, quantitativo e transversal, realizado em um hospital de ensino, referência para o pré-natal de alto risco do município de Uberaba (MG) e região, composta por 27 municípios, e para gestação de risco habitual das residentes do Distrito I (população estimada em 150 mil habitantes).

Participaram do estudo, gestantes a partir da $30^{\text {a }}$ semana gestacional, independente da idade (idade inferior a 18 anos foram autorizadas pelos responsáveis legais e assentido 0 consentimento). Foram excluídas, gestantes com malformações fetais e/ou que tiveram como desfecho aborto, óbito fetal ou natimorto. Assim, foram incluídas 208 gestantes que passaram por atendimento pré-natal no período entre 01 de dezembro de 2014 a 28 de fevereiro de 2015 , sendo a amostra determinada por conveniência.

Realizada entrevista por meio da aplicação de um instrumento construído pelos próprios autores, que foi testado mediante estudo-piloto com dez gestantes e estes instrumentos foram excluídos da análise. Não houve inclusão ou exclusão de variáveis após a aplicação do piloto, pois todas estavam adequadas para responder aos objetivos.

Foram coletadas informações sobre as variáveis: idade; se vivia com o companheiro; renda pessoal; ocupação; doenças; etilismo; tabagismo; uso de drogas ilícitas; idade gestacional no momento da entrevista; número de consultas pré-natal, gestações, filhos vivos e intercorrências na gestação.

Em relação à percepção sobre os diferentes tipos de parto, as gestantes foram questionadas se receberam orientação durante a gestação e qual profissional a orientou. Após foram questionadas: Qual tipo de parto mais seguro? Qual tipo de parto acredita que receba alta mais rápida da maternidade? Qual tipo de parto acha mais cômodo/confortável? Qual tipo de parto acha que o procedimento seja mais rápido? Qual tipo de parto acha que possui maior risco de morte para o bebê? Qual tipo de parto acha que possui maior risco de complicações? Foram fornecidas cinco possibilidades de resposta: parto normal; cesárea; fórceps, todos os tipos e, não sei. As variáveis foram analisadas por meio de estatística descritiva com o Statistical Package for the Social Sciences versão 23.0.

O estudo fez parte do projeto maior "Expectativas e desfechos em relação ao tipo de parto", aprovado pelo Comitê de Ética em Pesquisa da Universidade Federal do Triângulo Mineiro, parecer $n^{\circ} 814.120$ de 06 de agosto de 2014. Desta forma, o estudo respeitou as exigências formais contidas nas normas nacionais e internacionais regulamentadoras de pesquisas envolvendo seres humanos. 


\section{Caracterização da amostra}

A idade média das entrevistadas foi de 26,2 $\pm 6,4$, variando de 14 a 41 anos. Destas, $6,2 \%$ eram adolescentes e 10,6\% tinham idade superior a 35 anos. A maioria afirmou ter companheiro $(82,7 \%)$, não possuíam renda própria $(52,4 \%)$ e não exerciam atividade remunerada $(53,8 \%)$.

Sobre as condições de saúde, 5,8\% declararam fazer uso de bebida alcoólica; 7,2\% eram tabagista; apenas uma mulher referiu uso de droga ilícita; $24,5 \%$ relataram possuir alguma doença, sendo mais frequente as síndromes hipertensivas $(21,6 \%)$ e o hipotireoidismo $(11,8 \%)$.

Quanto aos dados obstétricos, as gestantes possuíam idade gestacional média de 33,8 $\pm 3,4$, variando de 30 a 40 semanas de gestação; realizaram em média 6,3 $\pm 2,4$ consultas prénatal, com frequência variando de uma a 14 consultas. O número de gestações variou de uma a nove, com média de 2,5 $\pm 1,5$ gestações; o número médio de filhos vivos foi de $1,1 \pm 1,2$, variando de zero a seis e 13,9\% relataram intercorrências, sendo mais comuns o trabalho de parto prematuro $(34,5 \%)$ e sangramentos vaginais $(24,1 \%)$.

\section{Percepção sobre os diferentes tipos de parto}

Quando questionadas se receberam orientação sobre os diferentes tipos de parto, 54,8\% relataram não ter recebido nenhum tipo de orientação. Quanto ao profissional que orientou, 0 médico foi o profissional mais citado (45,6\%), seguido pelo grupo de gestantes $(18,9 \%)$; enfermeiro (15,6\%) e equipe multiprofissional (3,3\%).

Em relação à percepção das gestantes sobre os diferentes tipos de parto, obtivemos que a maioria afirmou que o parto normal é mais seguro, recebia alta hospitalar mais rápida, é mais cômodo/confortável para a mulher, e, é o procedimento mais rápido; 47,1\% acreditavam que o parto fórceps possui maior risco de morte para o recém-nascido e a maioria relatou que a cesárea possui maior risco de complicações. Respostas acerca da percepção sobre os diferentes tipos de parto são apresentadas na Tabela I.

Tabela I - Frequência das variáveis de resposta das 208 gestantes entrevistadas sobre sua percepção acerca dos diferentes tipos de parto. Uberaba/MG, 2015.

\begin{tabular}{|c|c|c|}
\hline Variáveis & & $\mathbf{N}(\%)$ \\
\hline Qual tipo de parto mais seguro? & $\begin{array}{l}\text { Normal } \\
\text { Cesáreo } \\
\text { Todos os tipos } \\
\text { Não sabe/Não respondeu }\end{array}$ & $\begin{array}{l}168(80,8) \\
37(17,8) \\
2(1,0) \\
1(0,5)\end{array}$ \\
\hline $\begin{array}{l}\text { Qual tipo de parto acredita que receba alta mais rápida da } \\
\text { maternidade? }\end{array}$ & $\begin{array}{l}\text { Normal } \\
\text { Cesáreo } \\
\text { Não sabe/Não respondeu }\end{array}$ & $\begin{array}{l}206(99,0) \\
1(0,5) \\
1(0,5)\end{array}$ \\
\hline $\begin{array}{l}\text { Qual tipo de parto acha mais cômodo/confortável para a } \\
\text { mulher? }\end{array}$ & $\begin{array}{l}\text { Normal } \\
\text { Cesáreo } \\
\text { Não sabe/Não respondeu }\end{array}$ & $\begin{array}{l}147(70,7) \\
55(26,4) \\
6(2,9)\end{array}$ \\
\hline $\begin{array}{l}\text { Qual tipo de parto acha que o procedimento seja mais } \\
\text { rápido? }\end{array}$ & $\begin{array}{l}\text { Normal } \\
\text { Cesáreo } \\
\text { Todos os tipos } \\
\text { Não sabe/Não respondeu }\end{array}$ & $\begin{array}{l}118(56,7) \\
83(39,9) \\
1(0,5) \\
6(2,9)\end{array}$ \\
\hline $\begin{array}{l}\text { Qual tipo de parto acha que possui risco de morte para o } \\
\text { RN? }\end{array}$ & $\begin{array}{l}\text { Normal } \\
\text { Cesáreo } \\
\text { Fórceps } \\
\text { Todos os tipos } \\
\text { Não sabe/Não respondeu }\end{array}$ & $\begin{array}{l}30(14,4) \\
56(26,9) \\
98(47,1) \\
14(6,7) \\
10(4,8)\end{array}$ \\
\hline Qual tipo de parto acha que possui risco de complicações? & $\begin{array}{l}\text { Normal } \\
\text { Cesáreo } \\
\text { Fórceps } \\
\text { Todos os tipos } \\
\text { Não sabe/Não respondeu }\end{array}$ & $\begin{array}{l}29(13,9) \\
108(51,9) \\
36(17,3) \\
28(13,5) \\
7(3,4) \\
\end{array}$ \\
\hline
\end{tabular}


A maioria das gestantes entrevistadas realizou o pré-natal adequadamente, com média de aproximadamente seis consultas. Este número de consultas é considerado ideal segundo 0 Programa de Humanização no Pré-Natal e Nascimento, sendo indicadas uma consulta no primeiro, duas no segundo e três no terceiro trimestre gestacional [11]. Semelhantemente, estudo realizado no Rio Grande do Sul apontou que $73,7 \%$ das gestantes assistidas em um hospital universitário realizaram seis ou mais consultas [12], e em estudo realizado em João Pessoa, 90\% realizaram mais de seis consultas [13].

Embora tenham realizado o pré-natal adequadamente, menos da metade das gestantes referiu ter recebido orientações a respeito dos diferentes tipos de parto. De forma semelhante, estudo com 137 puérperas, apontou que 70,8\% referiram não ter sido orientada sobre os tipos de parto, independente dos serviços de atenção à saúde utilizados (público ou privado). As mesmas referiram que durante as consultas foram orientadas apenas sobre 0 exame físico obstétrico, resultados de exames laboratoriais e encaminhamentos necessários [12].

Estudo que avaliou a assistência pré-natal em um município brasileiro constatou que $90 \%$ realizaram o pré-natal adequadamente; $40 \%$ com início no primeiro trimestre, todas realizaram todos os exames laboratoriais básicos e tiveram cobertura antitetânica. Contudo, apenas $11 \%$ das gestantes participaram de alguma ação educativa durante a gestação, recebendo o menor escore de frequência entre todos os itens avaliados [13]. Dados semelhantes ao nosso estudo, em que apenas $18,9 \%$ frequentaram grupos de gestante.

Os dados apontam que entre as mulheres que receberam orientação sobre os tipos de parto, foram mais frequentes as orientações profissionais individuais durante as consultas. Ressalta-se que educação em saúde é importante ferramenta na assistência ao ciclo gravídicopuerperal e, enquanto prática durante o pré-natal, pode ser representada de diversas formas, sendo mais comuns palestras, grupos de gestantes e ações educativas individuais durante as consultas [14].

Os autores destacam as diferentes formas de educação em saúde. Palestras podem ser definidas como exposição de uma temática dialogada ou não. Grupos de gestantes representam boas estratégias pedagógicas de socialização de conhecimentos e construção de saberes em grupo e deve ser estimulada como prática educativa entre os enfermeiros. Já o momento da consulta seria o espaço legítimo, escolhido e ideal para as ações individualizadas. Contudo, ações educativas não devem ser restritas apenas a este momento, uma vez que a consulta de pré-natal envolve muitas atividades tais como: atividades assistenciais, exame físico, atividades gerenciais, preenchimento de formulários e cartão de pré-natal e sabe-se do excessivo número de atendimentos agendados para os profissionais, o que poderia reduzir ou abolir esta atividade durante a consulta [14].

Destaca-se também que não menos importante, a sala de espera torna-se espaço potencial para a educação em saúde. Trata-se de um espaço dinâmico no qual, enquanto aguarda-se a consulta, preenche-se o tempo ocioso com informações de interesse para a saúde da gestante, neonato, e todos que a estiverem acompanhando [15].

O acompanhamento pré-natal é momento propício para o profissional de saúde exercer importante influência sobre a mulher com papel relevante de educador em saúde. $O$ profissional deve oferecer apoio orientando a gestante em relação a todo o processo gestacional e seu desfecho, incluindo os diferentes tipos de parto, informações significativas para manter a mulher segura e preparada [16]. Além disso, a satisfação com a assistência prénatal apresenta relação direta com as orientações recebidas durante as consultas $[17,18]$.

Em nosso estudo, o médico foi o profissional mais citado como orientador sobre os tipos de parto; o enfermeiro foi o segundo mais citado, entretanto o percentual de citação foi de $15,6 \%$. É importante ressaltar que o enfermeiro deve assumir sua função na educação em saúde já que tem papel fundamental na promoção da saúde da mulher desde o início da gravidez, compondo a rede de apoio junto com seus familiares. Também apresenta importância no fortalecimento da capacidade da mulher para que resgate seu papel na condução da gravidez e parto, estimulando para que a mesma assuma o protagonismo do processo de nascimento, com consciência e segurança [18].

As limitações do estudo foram quanto ao método utilizado, por se tratar de estudo com desenho descritivo e transversal e no que tange a validade externa, uma vez que os dados não podem ser generalizados para outras realidades. Salientamos que a partir dos resultados 
encontrados poderão ser realizados novos estudos sobre a temática, que sejam comprovados por meio de testes de hipóteses ou que utilizem delineamentos diferentes.

Os dados apresentados no presente estudo possibilitaram delinear um perfil das gestantes que são atendidas no hospital de ensino, assim como detectar a necessidade de educação em saúde e orientação dos diferentes tipos de parto para esta população. A partir dos resultados, poderão ser instituídas orientações sobre o tema tanto na instituição que foi realizado o estudo quanto em outras instituições, dado que em comparação com outros estudos, identifica-se esta necessidade em diferentes contextos e instituições.

\section{Conclusão}

A maioria das gestantes acreditava que o parto normal é o mais seguro, possibilita alta hospitalar mais rápida da maternidade, consiste na via mais cômoda ou confortável para as mulheres e é o procedimento mais rápido para o nascimento. Menos da metade das entrevistadas acreditava que o parto fórceps possui risco de morte para o recém-nascido e cerca de metade das gestantes relatou que o parto cesárea possui maior risco de complicações para mãe e para o recém-nascido. Destaca-se que menos da metade das entrevistadas receberam orientações profissionais sobre os diferentes tipos de parto, suas vantagens e desvantagens.

Frente aos resultados e altos índices de cesáreas, identifica-se a necessidade de educação em saúde referente aos diferentes tipos de parto e ressalta-se a importância do enfermeiro neste processo, enquanto educador.

\section{Agradecimentos}

Apoio Financeiro - Bolsa de Iniciação Científica - FAPEMIG - Fundação de Apoio à Pesquisa em Minas Gerais.

1. Guerreiro EM, Rodrigues DP, Queiroz ABA, Ferreira MA. Educação em saúde no ciclo gravídico-puerperal: sentidos atribuídos por puérperas. Rev Bras Enferm 2014;67(1):13-21.

2. Jamas MT, Hoga LAK, Reberte LM. Narrativas de mulheres sobre a assistência recebida em um centro de parto normal. Cad Saúde Pública 2013;29(12):2436-46.

3. Leal MC, Pereira APE, Domingues RMSM, Theme Filha MM, Dias MAB, NakamuraPereira M, et al. Intervenções obstétricas durante o trabalho de parto e parto em mulheres brasileiras de risco habitual. Cad Saúde Pública 2014;30(Suppl1):S17-S32.

4. Organização Mundial de Saúde. Declaração da OMS sobre Taxas de Cesáreas. [Internet] 2015. [citado 2017 Jan 13]. Disponível em: http://apps.who.int/iris/bitstream/10665/161442/3/WHO_RHR_15.02_por.pdf?ua=1\&ua= 1

5. Betran AP, Torloni MR, Zhang J, Ye J, Mikolajczyk R, Deneux-Tharaux $\mathrm{C}$ et al. What is the optimal rate of caesarean section at population level? A systematic review of ecologic studies. Reprod Health 2015;12(1):57.

6. Domingues RMSM, Dias MAB, Nakamura-Pereira M, Torres JA, D'Orsi E, Pereira APE, et al. Processo de decisão pelo tipo de parto no Brasil: da preferência inicial das mulheres à via de parto final. Cad Saúde Pública 2014;30(Suppl1):S101-S116.

7. Datasus. Distribuição de nascimentos segundo tipo de parto. 2016. [Internet] 2018. [citado 2018 jul.17]. Disponível em: http://tabnet.datasus.gov.br/cgi/tabcgi.exe?sinasc/cnv/nvuf.def.

8. Torres JA, Domingues RMSM, Sandall J, Hartz Z, Gama SGN, Theme Filha MM, et al. Cesariana e resultados neonatais em hospitais privados no Brasil: estudo comparativo de dois diferentes modelos de atenção perinatal. Cad Saúde Pública 2014;30(Suppl 1):S220-S231.

9. Almeida NAM, Medeiros M, Souza MR. Perspectives of normal delivery pain of primigravid during the antenatal period. Texto Contexto Enferm 2012;21(4):819-27.

10. Velho MB, Santos EKA, Collaço VS. Parto normal e cesárea: representações sociais de mulheres que os vivenciaram. Rev Bras Enferm 2014;67(2):282-9. 
11. Ministério da Saúde (BR). Secretaria de Atenção à Saúde. Departamento de Atenção Básica. Atenção ao pré-natal de baixo risco/Série A. Normas e Manuais Técnicos. Cadernos de Atenção Básica n 32. Brasília: Ministério da Saúde, 2012.

12. Barbieri A, Fonseca LM, Ceron MI, Fedosse E. Análise da atenção pré-natal na percepção de puérperas. Distúrb Comum 2012;24(1):29-39.

13. Silva EP, Lima RT, Ferreira NLS, Carvalho e Costa MJ. Pré-natal na atenção primária do município de João Pessoa-PB: caracterização de serviços e usuários. Rev Bras Saude Mater Infant 2013;13(1):29-37.

14. Guerreiro EM, Rodrigues DP, Queiroz ABA, Ferreira MA. Educação em saúde no ciclo gravídico-puerperal: sentidos atribuídos por puérperas. Rev Bras Enferm 2014;67(1):13-21.

15. Santos DS, Andrade ALA, Lima BSS, Silva YN. Sala de espera para gestantes: uma estratégia de educação em saúde. Rev Bras Educ Méd 2012;36 (Supl2):62-7.

16. Silva SPC, Prates RCG, Campelo BQA. Parto normal ou cesariana? Fatores que influenciam na escolha da gestante. Rev Enferm UFSM 2014;4(1):1-9.

17. Jorge HMF, Hipólito MCV, Masson VA, Silva RM. Prenatal care and public policies for women's health: integrative review. Rev Bras Promoç Saúde 2015;28(1):140-8.

18. Luz NF, Assis TR, Rezende FR. Puérperas adolescentes: percepções relacionadas ao pré-natal e ao parto. ABCS Health Sci 2015;40(2):80-4. 